\title{
BUDD-VANNIMENUS THEOREM AND WORK FUNCTION EXPRESSION FOR A METAL-METALLIC ADLAYER SYSTEM* ${ }^{*}$
}

\author{
J. PeiserT \\ Institute of Physics, Technical University of Wroctaw \\ Wybrzeże Wyspiańskiego 27, 50-370 Wroclaw, Poland \\ AND K.F. WOJCIECIIOWSKI \\ Institute of Experimental Plyssics, Wroclaw University \\ Cybulskiego 36, 50-205 Wroclaw, Poland \\ (Received March 1, 1994; in final form April 21, 1994)
}

\begin{abstract}
The generalization of the Budd-Vannimenus theorem for jellium model of metal surface, when the positive background is described by a non-negative function, is given. Using the displacement electron density profile method and the generalized Budd-Vannimenus theorem it is shown that work function expressions given on the basis of Koopmans theorem and by Monnier et al. are equivalent both for a clean metal and for a metal covered by a metallic adlayer.
\end{abstract}

PACS numbers: $71.10 .+\mathrm{x}, 73.10 . \mathrm{Jn}_{\mathrm{n}}$

\section{Introduction}

The original Budd-Vannimenus theorem (BVT) [1] relatcs the difference in electrostatic potential at the metal surface and that in the bulk, to the energy per electron for the uniform electron gas. Thus information about surface properties is gained through the well-understood properties of bulk matter. The first generalization of BVT was given by Bigun [2] for the case of jellium with the positive background

$$
n_{+}(x)=\bar{n} \Theta(-x)+\bar{n}_{\mathrm{a}} \Theta(x) \Theta(-x+d)
$$

(semi-infinite metal + metallic adlayer of thickness $d), \Theta$ is the ordinary step function. The second one, proposed by Monnier and Perdew [3], introduces the additional external potential simulating the periodicity of the metal interior.

*This research was supported in part (K.F.W.) by the Wroctaw University grant 2016/W/IFD/92. 
The BVT often was used as a constraint set on the electrostatic potential in order to determine variational parameters appearing in trial electron density profile and then to calculate the surface energy and work function [2, 4]. For the work function calculation the so-called Koopmans (K) definition [5] as well as the "displacement-profile-change-in-self-consistent-field" (DP $\triangle \mathrm{SCF} \equiv \mathrm{D}$ ) expression [6] was used.

In Sec. 2 we present the generalization of the BVT for jellium model, when the positive background is described by a non-negative function. In Sec. 3 we show, using D-method, that work function expression given on the ground of Koopmans theorem, $W_{\mathrm{K}}$, and by Perdew and Salini [6], $W_{\mathrm{D}}$, are equivalent as well for the clean metal as for that covered by metallic adlayer, when the BVT is fulfilled.

\section{Gencralization of the Budd-Vannimenus theorem}

Now we shall give the generalization of the BVT when $n_{+}(x)$, the positive charge density, is such that

$$
n_{+}( \pm \infty)=0
$$

$n_{+}(x)$ changes only in the neighbourhood of $x= \pm L / 2$ and

$$
\int n_{+}(x) \mathrm{d} x=N=\text { const }<\infty,
$$

where $L$ denotes the thickness of the slab having the area $A$.

The infinitesimal stretching of $n_{+}$, conserving the above integral, will be

$$
n_{+}(x) \rightarrow n_{+}^{\prime}(x)=(1-\lambda) n_{+}(x-\lambda x) \equiv n_{+}(x)+\Delta n_{+}(x)
$$

and

$$
\Delta n_{+}(x)=-\lambda\left[n_{+}(x)+x \frac{\mathrm{d} n_{+}(x)}{\mathrm{d} x}\right],
$$

to the first order in $\lambda \equiv \Delta L / L$.

According to the IIellman-Feymman theorem [7] the change $\Delta E$ in the total energy of the system upon a small change $\Delta n_{+}(r)$ is given by

$$
\Delta E=-\int \mathrm{d} r \phi(r) \Delta n_{+}(r),
$$

where $\phi$ denotes the electrostatic potential. Combining Eq. (5) with Eq. (6) yields*

$$
\Delta E / A \approx \lambda L \bar{n} \phi(0)+\lambda \int_{-\infty}^{\infty} x \frac{\mathrm{d} n_{+}(x)}{\mathrm{d} x} \phi(x) \mathrm{d} x,
$$

where

$$
\bar{n}=N / L .
$$

Recalling that the derivative $\mathrm{d} n_{+} / \mathrm{d} x$ is non-zero only in the vicinity of $x= \pm L / 2$, the integral in Eq. (7) may be transformed to the form $\lambda L \int_{0}^{\infty} \Theta(x) \frac{\mathrm{d} n_{+}}{\mathrm{d} x} \phi(x) \mathrm{d} x$. Now let us move the second slab surface as well as the slab center to the minus

*Atomic units are used $\left(\hbar=|e|=m_{\mathbf{e}}=1\right)$. 
infinity. Following the proof of Monnier and Perdew [3] (Appendix F therein) we get the BVT

$$
\bar{n} \frac{\mathrm{d} \varepsilon_{\mathrm{T}}(\bar{n})}{\mathrm{d} \bar{n}}+\frac{C n(X)}{\bar{n}}=-\phi(-\infty)-\frac{1}{\bar{n}} \int_{-\infty}^{\infty} \frac{\mathrm{d} n_{+}(x)}{\mathrm{d} x} \phi(x) \mathrm{d} x,
$$

where $\varepsilon_{\mathrm{T}}=\frac{3}{10} k_{\mathrm{F}}^{2}+\varepsilon_{\mathrm{xc}}$ is the sum of the average kinetic, exchange and correlation energies of uniform electron gas per particle. $C$ and $X$ denote the variational parameters of Monnier and Perdew potential $V(x)=C \Theta(-x+X)$ and $n(x)$ describes the electron density profile in the direction perpendicular to the considered system. For $n_{+}$being the $k$-step function

$$
n_{+}(x)= \begin{cases}\bar{n}_{0} & \text { if } x<d_{0}, \\ \bar{n}_{i} & \text { if } d_{i-1}<x<d_{i} \\ \bar{n}_{k}=0 & \text { if } d_{k-1}<x\end{cases}
$$

where $i=1,2, \ldots, k-1$, we have (with $\delta(x)$ being the Dirac function)

$$
\frac{\mathrm{d} n_{+}(x)}{\mathrm{d} x}=\sum_{i=0}^{k-1}\left(\bar{n}_{i+1}-\bar{n}_{i}\right) \delta\left(x-d_{i}\right),
$$

and the formula (9) takes the form

$$
\bar{n}_{0} \frac{\mathrm{d} \varepsilon_{\mathrm{T}}\left(\bar{n}_{0}\right)}{\mathrm{d} \bar{n}_{0}}+C \frac{n(X)}{\bar{n}_{0}}=-\frac{1}{\bar{n}} \sum_{i=0}^{k-1}\left(\bar{n}_{i+1}-\bar{n}_{i}\right) \phi\left(d_{i}\right)-\phi(-\infty) .
$$

Expression (12) for $k=2$ exhibits the Bigun result [2].

The above generalization of BVT can be used to many surface problems for which positive background may be simulated by the function given by Eq. (10), thus for instance, to layer on layer adsorption or to surface segregation. In the next section we use it to examine the relation between $K$ and $D$ expressions for work function.

\section{Work function expression}

The first commonly used definition of work function is reminiscent of Koopmans theorem [5] for removal energy of an electron from an atom, and has the following form [8]:

$$
W_{\mathrm{K}}=\Delta \phi-\bar{\mu}=\Delta \phi-\frac{3}{10} k_{\mathrm{F}}^{2}-\varepsilon_{\mathrm{xc}}-\frac{\mathrm{d} \varepsilon_{\mathrm{T}}(\bar{n})}{\mathrm{d} \bar{n}},
$$

where $\Delta \phi$ is the surface dipole barrier $[8,9]$, and $k_{F}-$ the Fermi momentum. The electrostatic potential $\phi(x)$, connected with the total-charge-density profile $n_{\mathrm{T}}(x)$ by the Poisson equation has the form

$$
\phi(x)=\phi(-\infty)+4 \pi \int_{-\infty}^{x} \mathrm{~d} x^{\prime}\left(x^{\prime}-x\right) n_{\mathrm{T}}\left(x^{\prime}\right)
$$

where $x$-axis is perpendicular to the surface of metal-adsorbate system and $n_{\mathrm{T}}(x)=$ $n(x)-n_{+}(x)$. Here $n(x)$ denotes the electron density profile of metal (with bulk density $\bar{n}$ ) and $n_{+}(x)$ is arbitrary non-negative function such that the conditions 
$n_{+}(-\infty)=\bar{n}$ and $n_{+}(\infty)=0$ are satisfied. The $\mathrm{D}$ expression for work function is [6]:

$$
W_{\mathrm{D}}=\phi(\infty)-\phi(0)-\frac{3}{10} k_{\mathrm{F}}^{2}-\varepsilon_{\mathrm{xc}}+\frac{1}{\bar{n}} \int_{-\infty}^{\infty} \delta v(x) \frac{\mathrm{d} n(x)}{\mathrm{d} x} \mathrm{~d} x
$$

where $\delta v(x)$ is the planar average of the discrete lattice potential (for the jellium model $\delta v(x) \equiv 0)$.

Let us evaluate now $\phi_{\text {es }}$, the electrostatic contribution to work function (see [10], Appendix D) employing the change-in-self-consistent-field ( $\triangle \mathrm{SCF})$ method for the positive jellium density $n_{+}(x)$ assumed above. Denoting $n_{\Sigma}(x)$ the electron density profile of the metal with $\Sigma$ electrons per unit area added on its surface and with $-\Sigma$ charge in the infinity, one can do the replacement

$$
\begin{aligned}
& n_{\Sigma}(x)=n^{\prime}(x)+\delta(x-\infty) \Sigma, \\
& n^{\prime}(x)=n_{\Sigma=0}\left(x+\frac{\Sigma}{\bar{n}}\right) .
\end{aligned}
$$

Since $\Delta$ SCF method expresses work function as

$$
\phi=\frac{\mathrm{d} \sigma_{\mathrm{es}}}{\mathrm{d} \Sigma} \|_{\Sigma=0},
$$

( $\sigma$ denotes the surface energy) our task is to evaluate the derivative of the electrostatic part $\sigma_{\mathrm{es}}$ of the surface energy with respect to the surface charge density $\Sigma$, or to find the linear coefficient of the power expansion of $\sigma_{\mathrm{es}}$ with respect to the parameter $\Delta \equiv \Sigma / \bar{n}$. Using the definition

$$
\sigma_{\mathrm{es}}=\frac{1}{2} \int_{-\infty}^{\infty} \mathrm{d} x \phi\left(\left[n_{\Sigma}\right] ; x\right)\left[n_{\Sigma}(x)-n_{+}(x)\right],
$$

the electrostatic energy becomes, to the first order in $\Delta$

$$
\sigma_{\mathrm{es}}=\frac{1}{2} \int_{-\infty}^{\infty} \mathrm{d} x \phi\left(\left[n_{\Sigma}\right] ; x\right)\left[n^{\prime}(x)-n_{+}(x)\right]+\frac{1}{2} \bar{n} \phi\left(\left[n_{0}\right] ; \infty\right) \Delta,
$$

where $n_{0}=n_{\Sigma=0}$. Employing Eq. (14) and using Eq. (17) one can find that

$$
\phi\left(\left[n_{\Sigma}\right] ; x\right)=\phi\left(\left[n_{0}\right] ; x+\Delta\right)+4 \pi \Delta \int_{-\infty}^{\infty} \mathrm{d} x^{\prime}\left(x^{\prime}-x\right) \frac{\mathrm{d} n_{+}\left(x^{\prime}\right)}{\mathrm{d} x^{\prime}}
$$

and obtain

$$
\begin{aligned}
& \sigma_{\mathrm{es}} \approx \frac{1}{2} \int_{-\infty}^{\infty} \mathrm{d} x \phi\left(\left[n_{0}\right] ; x+\Delta\right)\left[n_{0}(x+\Delta)-n_{+}(x+\Delta)\right. \\
& +\left[\int_{-\infty}^{\infty} \mathrm{d} x \phi\left(\left[n_{0}\right] ; x\right) \frac{\mathrm{d} n_{+}(x)}{\mathrm{d} x}+\frac{1}{2} \phi\left(\left[n_{0}\right] ; \infty\right)\right] \Delta .
\end{aligned}
$$

Now using Eq. (18) we arrive at the expression

$$
\phi_{\mathrm{es}} \equiv \frac{\mathrm{d} \sigma_{\mathrm{es}}}{\mathrm{d} \Sigma} \|_{\Sigma=0} \approx \phi(\infty)+\frac{1}{\bar{n}} \int_{-\infty}^{\infty} \mathrm{d} x \phi(x) \frac{\mathrm{d} n_{+}(x)}{\mathrm{d} x},
$$

and finally

$$
W_{\mathrm{D}}=\frac{3}{10} k_{\mathrm{F}}^{2}-\varepsilon_{\mathrm{xc}}+\int_{-\infty}^{\infty} \mathrm{d} x \delta v(x) \frac{\mathrm{d} n(x)}{\mathrm{d} x}+\phi(\infty)+\frac{1}{\bar{n}} \int_{-\infty}^{\infty} d x \phi(x) \frac{\mathrm{d} n_{+}(x)}{\mathrm{d} x}
$$


Employing the generalized Budd and Vannimenus theorem given by Eq. (9), for variational potential $V(x) \equiv 0$,

$$
-\bar{n} \frac{\mathrm{d} \varepsilon_{\mathrm{T}}(\bar{n})}{\mathrm{d} \bar{n}}=\frac{1}{\bar{n}} \int_{-\infty}^{\infty} \mathrm{d} x \phi(x) \frac{\mathrm{d} n_{+}(x)}{\mathrm{d} x}+\phi(-\infty),
$$

we arrive at the equality $W_{\mathrm{K}}=W_{\mathrm{D}}$ for any $n_{+}(x)$. For clean metal model $\left(n_{+}(x) \equiv\right.$ $\bar{n} \Theta(-x))$ this equivalence has been shown carlier by Mahan and Schaich [11]. It is to be noted that the Bigun result (with $n_{+}$given by Eq. (1))

$$
\phi(0)-\phi(-\infty)+\frac{\bar{n}_{\mathrm{a}}}{\bar{n}}[\phi(d)-\phi(0)]=\bar{n} \frac{\mathrm{d} \varepsilon_{\mathrm{T}}(\bar{n})}{\mathrm{d} \bar{n}},
$$

is valid also when $\bar{n}_{\mathrm{a}} / \bar{n} \geq 1$.

\section{References}

[1] H.F. Budd, J. Vannimenus, Plyys. Rev. Letl. 31, 1218 (1973); 31, 1430(E) (1973).

[2] G.I. Bigun, Ukr. Fiz. Zh. 24, 1313 (1979) (in Russian); Fiz. Elektronika 18, 114 (1979) (in Russian).

[3] R. Monnier, J.P. Perdew, Pliys. Rev. B 17, 2595 (1978); 22, 1124 (E) (1980).

[4] V. Sahni, C.Q. Ma, Phys. Rev. B 22, 5987 (1980) and references therein.

[5] T. Koopmans, Physica (Nelherlands) 1, 104 (1933).

[6] J.P. Perdew, V. Salıni, Solid Slale Commun. 30, 87 (1979).

[7] H. Hellman, Einfultrung in die Quantenchemie, Deuticke, Leipzig 1973; R.P. Feynman, Phys. Rev. 56, 340 (1939); sce also B.M. Dem, Rev. Mod. Phys. 45, 22 (1973).

[8] N.D. Lang, W. Kohn, Phys. Rev. B 3, 1215 (1971).

[9] N.D. Lang, Solid Slate Phys. 28, 225 (1973).

[10] R. Monnier, J.P. Perdew, D.C. Langreth, J.W. Wilkins, Phys. Rev. B 18, 656 (1978).

[11] G.D. Mahan, W.L. Schaich, Phys. Rev. B 10, 2647 (1974). 